ice, two, three or four. But if ye fear ye shall not be able to deal justly (with them) then only one (IV: 3) . . YYe are never able to be fair and just as between women, even if it is your ardent desire (IV: 129)" (S. 110 f.). Daß der Koran die Polygamie trotzdem, wenn auch unter Einschränkungen, beibehalten habe, liege an den sozio-ökonomischen Erfordernissen der Zeit, welche unter anderem eine Versorgung der durch die zahlreichen Kriege hinterbliebenen Witwen und Waisen notwendig gemacht hätten. Mit dem Wandel der gesellschaftlichen und wirtschaftlichen Gegebenheiten und Bedürfnisse sei auch eine Rückkehr zu der Grundentscheidung des Koran zugunsten der Monogamie möglich und angezeigt.

Espositos Buch behandelt interessante Dimensionen einer Dynamik und Anpassungsfähigkeit des islamischen Rechts. Das Problem dürfte indes in der häufig stärkeren Position der Traditionalisten liegen, die der historischen Sichtweise Espositos nicht unbedingt folgen werden. Einen Überblick über den derzeitigen Meinungsstand hierzu zu geben war offensichtlich nicht sein Ziel. Ob er seinen Anspruch, Methoden zu entwickeln, die auch von den Traditionalisten akzeptiert werden, erfüllen konnte, bleibt abzuwarten. Anders als von der Formulierung des Titels her zu erwarten ist, liegt der Schwerpunkt von Espositos Ausführungen nicht auf der Stellung der Frau, sondern auf der Methodik der Rechtsreform. Dieser Thematik widmet er sich mit einer gewissen aus seinen Ausführungen sprechenden Begeisterung, während die Situation der Frau eher der bald in den Hintergrund tretende Aufhänger zu sein scheint. Bezeichnenderweise klärt er weder im Vorwort noch an anderer Stelle, was er unter der "Frauenfrage" versteht und wie er den von ihm laut Titel zu behandelnden Gegenstand "Frauen im islamischen Familienrecht" abgrenzt. Auch die Auswahlbibliographie am Ende des Buches enthält kaum Titel, die sich ausdrücklich mit der Stellung der Frau befassen.

Sieht man von einer gewissen Enttäuschung der durch den Buchtitel geweckten Erwartungen ab, so ist Espositos Buch ein interessanter Beitrag zur Modernisierungsproblematik des islamischen Rechts.

Ulrike Wanitzek

\title{
Albert Wirtz
}

Krieg in Afrika. Die nachkolonialen Konflikte in Nigeria, Sudan, Tschad und Kongo Franz Steiner Verlag, Wiesbaden, 1982

Die Studie von A. Wirz, einem Schweizer Historiker, befaßt sich mit einem bestimmten Typus von Krieg, dem Bürgerkrieg, geht aber in ihrem Anspruch weit über die Analyse des Kriegsgeschehens im engeren Sinne und seiner Ursachen hinaus. Dieses wird im größeren Zusammenhang der Kolonisation samt ihren intendierten und nicht intendierten Auswirkungen sowie der anschließenden Dekolonisation und dem damit verbundenen mehr oder weniger erfolgreich verlaufenden Staatsbildungsprozeß gesehen, also in einen 
mehrere Jahrzehnte umspannenden historischen Rahmen eingefügt. Um in seinen Erkenntnissen nicht von historischen Singularkonstellationen abhängig zu sein, hat sich der Autor der arbeitsreichen Aufgabe unterzogen, nicht weniger als vier Fälle inneren Krieges zu untersuchen (allerdings werden die Kriterien der getroffenen Fallauswahl an keiner Stelle näher expliziert). Bei dem aufwendigen Vorhaben geht es ihm vor allem darum, simplifizierenden Erklärungen entgegenzutreten, etwa jener, die kriegerischen Konflikte ließen sich aus dem traditionellen af rikanischen Tribalismus ableiten, der nach der Unabhängigkeit alsbald durch die schüttere demokratische Fassade wieder hindurchgebrochen sei, oder sie seien durch die im Verlauf der Modernisierung und Integration Afrikas in die kapitalistische Weltwirtschaft entstandenen Klassenspannungen bedingt. Beide Ansätze greifen nach Wirz zu kurz, vielmehr sei von einer Uberlagerung der ursprünglichen ethnischen Identitäten durch Schichtstrukturen und somit von ihrer gegenseitigen Brechung und Verklammerung auszugehen, wobei der Differenzierung zwischen ländlichem und städtischem Sozialmilieu erhebliche Bedeutung zukomme. Das Mißtrauen gegenüber einfachen Erkärungsschemata, die zudem in durchsichtiger Weise ideologisch gefärbt seien, hält den Verfasser indessen nicht davon ab, nach Theorien Ausschau zu halten, die etwas zur Erhellung der kriegerischen Vorgänge in den vier afrikanischen Staaten beitragen könnten. Nutzen meint er insbesondere aus drei Theoriesträngen ziehen zu können: 1) den Zusammenbruchs- und Ungleichgewichtstheorien, deren soziologischer Stammvater E. Durkheim ist und die eine vorläufig abschließende Behandlung in T. E. Gurrs Theorien von der relativen Deprivation erfahren haben; 2) aus der Solidaritäts- und Organisationstheorie der Tillys zur Entstehung kollektiver Gewalt; 3) schließlich aus den Erkenntnissen der sog. Pluralismus-Schule (J. S. Furnivall, M. G. Smith u. a.), die sich auf die Möglichkeiten der Integration und die Gefahren der Dissoziation in multi-ethnischen Gesellschaften beziehen.

In den eigentlichen Fallstudien kommen diese theoretischen Gesichtspunkte allerdings nur äußerst selten zum Tragen. In ihnen wird aufs eingehendste die Entwicklung von Wirtschaft, Gesellschaft und Politik in den vier Staaten über die verschiedenen Phasen hinweg verfolgt. Wirz löst das eingangs erhobene Postulat einer differenzierend historischen Betrachtungsweise der Gewaltauseinandersetzungen in Afrika voll ein: Die Lage der vielfältigen ethnischen Gruppierungen und sozialen Schichten in den Großstädten und im Hinterland wird von ihm ebenso detailliert und genau - soweit es die vorhandene Sekundärliteratur erlaubte; in Einzelfällen scheute der Verfasser nicht vor der ErschlieBung neuen Quellenmaterials zurück - dargestellt wie der Werdegang der diversen politischen Bewegungen und Parteien. Weder fehlt es an einer sachkundigen Erläuterung der militärtechnischen Voraussetzungen und Erfolgschancen der jeweiligen Kampfhandlungen noch wird versäumt, das internationale Feld möglicher und realer Bündnispartner für die Aufständischen sowie die Beeinflussung ihrer Lage durch externe Waffenlieferungen und sonstige Hilfeleistungen aufzuzeigen. Der Rezensent gesteht, daß er nicht alle vier Fälle mit gleicher Intensität studiert hat. Nicht weil er die Lektüre nicht anregend, streckenweise sogar spannend fand - als Nichthistoriker fiel es ihm jedoch bisweilen schwer, über der Vielzahl der sorgfältig herauspräparierten Teilentwicklungen den Be- 
zug zum Gewaltgeschehen herauszufinden, eine hinlänglich deutliche Trennlinie zwischen strukturellen Wandlungsprozessen und ereignisgeschichtlichem Beiwerk zu erspähen. Wirz erschien eine solche Trennung im fallgeschichtlichen Teil offenkundig als verfrüht. Sein Anliegen war, dem Leser in breiter Form die Besonderheit der historischen Prozesse und politisch-gesellschaftlichen Verwicklungen in den einzelnen Ländern vor Augen zu führen, bevor er sich im Schlußteil an den Versuch einer generalisierenden Interpretation heranwagte. Insofern lassen sich die den eigentlichen Hauptteil des Buches ausmachenden Länderstudien auch als selbständige Monographien lesen und empfehlen. Die im letzten Kapitel behutsam gezogenen Schlußfolgerungen bestätigen teilweise Erkenntnisse der allgemeinen Guerilla- und Bürgerkriegsforschung, teils spiegeln sie die spezifischen Bedingungen innerer Kriege in Afrika. So überrascht es etwa denjenigen, der mit den Minderheitenproblemen in Europa vertraut ist, nicht, wenn Wirz feststellt, die Kongruenz von ethnischen und sozialen Konfliktfronten erhöhe die Wahrscheinlichkeit kollektiver Gewaltausbrüche, oder wenn er den Aufständischen besonders gute Erfolgschancen einräumt, sofern sie von außen mit Waffen und Finanzmitteln unterstützt würden und ihnen ein externer Flucht- und Regenerierungsraum in Form der nahen Grenze zu einem mit ihnen sympathisierenden Land zur Verfügung stünde. Auch seine Beobachtung, nicht die Kombattanten, sondern die Zivilbevölkerung seien die Hauptleidtragenden der Gewalt, läßt sich durch Guerillakriege in anderen Großregionen (etwa gegenwärtig in Zentralamerika) bestätigen; desgleichen wenn er das Fazit zieht, ungeachtet der revolutionären Parolen, die zuweilen zur Rechtfertigung der Aufstände dienten, würden diese nur selten an den Grundfesten der bestehenden Sozial- und Staatsordnung rütteln, verfolgten vielmehr meist das Ziel eines verbesserten Zugangs bestimmter gesellschaftlicher Sektoren zu wirtschaftlichen Pfründen und politischen Machtpositionen. Bei anderen Punkten findet dagegen das Spezifikum afrikanischer Sozialstrukturen einen deutlicheren Niederschlag, beispielsweise, wenn auf das Fortleben ethnischer Solidaritätsbindungen im urbanen Kontext hingewiesen wird oder auf die Persistenz herkömmlicher Verwandtschaftsmuster und Gemeinschaftsformen auf dem Lande, unabhängig von dessen Durchdringung durch kapitalistische Produktionsmethoden. Eines der Hauptverdienste der Arbeit besteht schließlich darin, die ambivalente Rolle der europäischen Kolonialmächte aufgezeigt zu haben, die einerseits relativ willkürlich neue politische und administrative Grenzen zogen, andererseits jedoch - dies gilt vor allem für die Briten - nach Möglichkeit den ethnischen und kulturellen Gegebenheiten Rechnung zu tragen suchten; die den modernen Nationalstaat und das Prinzip rationaler Verwaltung einführten, zugleich aber durch die autokratische Form ihrer Herrschaft und die konsequent durchgehaltene Rassentrennung zum Scheitern des kurzfristigen Demokratieexperiments nach ihrem Abzug und zum Wiederaufbrechen ethnischer Zwiste beitrugen.

Peter Waldmann 